\title{
Diagnosis of complicated FEVR preoperatively and intra-/post-operatively: characteristics and risk factors for diagnostic timing
}

\author{
Fengjie Xia, Jiao Lyu, Ping Fei and Peiquan Zhao*
}

\begin{abstract}
Background: To delineate the characteristics of complicated familial exudative vitreoretinopathy (FEVR) patients diagnosed before surgery or intra-/post-operatively and to analyze the risk factors for the diagnostic timing.

Methods: Forty-eight patients who underwent surgery and were diagnosed as FEVR in our department were retrospectively reviewed. Data were collected including the demographic and clinical characteristics of these patients. FEVR patients were divided into 2 groups according to the diagnostic timing: FEVR diagnosed pre-operatively (23 patients), FEVR diagnosed intra-/post-operatively (25 patients). Multivariable analysis was applied for analyzing the risk factors for diagnostic timing.

Results: The clinical characteristics of the FEVR patients were of great variability, including retinal detachment (RD), disappear of anterior chamber, retrolental membrane, epiretinal membrane (ERM), vitreous hemorrhage (VH), myopic foveoschisis (MF), lamellar macular hole (LMH), high myopia (HM). And the referral diagnosis or pre-operative diagnosis were always non-specific. The majority of the referral or preoperative diagnosis were unilateral RD $(52.1 \%)$, bilateral $\mathrm{RD}$ (8.3\%), unilateral persistent fetal vasculature (PFV) (8.3\%), bilateral PFV (4.2\%). There are two risk factors for the complicated FEVR cases diagnosed as FEVR preoperatively: pre-operative ocular manifestations with RD only (OR, 0.104; $p$-value, 0.022), positive parent's fluorescein angiography (FA) (OR, 0.105; p-value, 0.035).

Conclusions: The phenotypes of FEVR were greatly variable, they can mimic many non-specific vitreoretinal disorders. The most non-specific referral diagnosis/pre-operative diagnosis was unilateral RD, bilateral RD, unilateral PFV, bilateral PFV. A positive family history or a simple ocular presentation with RD only could contribute to diagnose FEVR preoperatively.
\end{abstract}

Keywords: Familial exudative vitreoretinopathy, Diagnosis timing, Risk factor, Clinical characteristic

\section{Background}

Familial exudative vitreoretinopathy (FEVR) is an inheritable vitreoretinal disease which is caused by anomalous and incomplete retinal angiogenesis. Clinically, FEVR is characterized by avascular peripheral retina, excessive vessel branchings, increased straightening vascular branching, vitreous adherence, retinochoroidal degeneration, macular

\footnotetext{
* Correspondence: zhaopeiquan@xinhuamed.com.cn

Department of Ophthalmology, Xinhua Hospital, Affiliated to Medicine

School of Shanghai Jiaotong University, No. 1665, Kongjiang Road, Shanghai 200092, China
}

(c) The Author(s). 2019 Open Access This article is distributed under the terms of the Creative Commons Attribution 4.0 International License (http//creativecommons.org/licenses/by/4.0/) which permits unrestricted use, distribution, and reproduction in any medium, provided you give appropriate credit to the original author(s) and the source, provide a link to the Creative Commons license, and indicate if changes were made. The Creative Commons Public Domain Dedication waiver (http://creativecommons.org/publicdomain/zero/1.0/) applies to the data made available in this article, unless otherwise stated.

and disc dragging, retinal exudation, radial retinal folds, and exudative, and/or tractional retinal detachments (RD) [1]. The expressivity of FEVR can be asymptomatic or with greatly variable [2]. FEVR staging system, presented by Pendergast and Trese in 1998, were widely used in the clinical research [3]. The staging of bilateral eyes in the same individual can be greatly asymmetric, such as one eye being stage 1-2 with no symptom, while the contralateral eye being stage 3-5 with RD [4]. Additionally, the disease course of FEVR was greatly skipped and unpredictable. It can be stable in stage 1-2 in the lifetime or be rapidly progressive from stage $1-2$ to stage $3-5$. Therefore, early diagnosis of 
FEVR is important and it is essential for FEVR patients to undergo lifelong regular follow-up [5].

However, in the clinic, FEVR is always mistaken for non-specific vitreoretinopathies and the diagnosis of FEVR is easily missed. Ranchod et.al reported that $71.7 \%$ FEVR patients were referred to their institution with a nonspecific diagnosis [4]. Because the manifestations of FEVR eyes are of greatly diverse form, such as RD, leukocoria, disappearance of anterior chamber, $\mathrm{VH}[6,7]$. Besides, due to the peripheral retinochoroidal scars of photocoagulation or cryotherapy, the diagnosis of FEVR in patients who had prior vitreoretinal surgery would be more challenging. Since FEVR is an inherited retinopathy, missed diagnosis of FEVR is not conducive to the patient's lifetime follow-up, and do not contribute to genetic counseling for those of child-bearing age. Many prior clinical studies have reported the clinical characteristics of untreated FEVR patients and surgery outcomes of FEVR-RD patients $[4,8-$ 11]. However, there are few studies reported the diagnostic timing of FEVR patients who underwent surgery.

In the study, we retrospectively analyzed 48 FEVR patients who underwent surgery and diagnosed as FEVR finally from March 2010 to May 2018 in Xinhua hospital. In the cohort, the diagnostic timing of FEVR patients was variable, including preoperatively, during the operation and in the follow-up of post-operation. We collected the demographic features, clinical characteristics of the cohort and their first surgery in our department. Furtherly, we analyzed the risk factors for diagnostic timing of the complicated FEVR patients. It would contribute to ophthalmologists to diagnose FEVR much earlier and prevent missed diagnosis of FEVR.

\section{Methods}

\section{Patients}

The retrospective study was conducted in accordance with the tenets of the Declaration of Helsinki and was approved by the Ethics Committee of Xinhua Hospital affiliated to Shanghai Jiao Tong University School of Medicine, Shanghai, China. All patients provided an informed consent prior to participating into the study. A total of 48 patients with FEVR who underwent surgery were studied. The 48 patients were diagnosed as FEVR in our department between March 2010 and May 2018. We found that these FEVR patients had one of the following findings in at least one eye: $\mathrm{RD}$, disappear or shallow of anterior chamber, retrolental membrane, leukocoria, ERM, vitreous hemorrhage (VH), myopic foveoschisis (MF). Among those ocular findings, $\mathrm{RD}$ is the most common ocular manifestations occurred in these FEVR patients. In the study, except RD, the rest of ocular findings as mentioned above were defined as other ocular manifestations. The clinical diagnostic criteria for FEVR were: (1) a lack of peripheral retinal vascular development, (2) full-term or preterm birth with a disease course not consistent with retinopathy of prematurity, and (3) variable degrees of non-perfusion, vitreoretinal traction, sub-retinal exudation, or retinal neovascularization occurring at any age. FEVR was classified according to the clinical staging criteria described by Pendergast and Trese. The inclusion criteria for the 48 FEVR patients in this study were as follows: (1) all patients underwent surgery in our department, (2) the diagnosis of FEVR was confirmed by careful peripheral fundus examination, positive findings in patient FA or positive family history. Patients were excluded if the diagnosis of FEVR was questionable (i.e., Unilateral FEVR that have negative genetic sequencing and no family history were excluded). Patients were excluded if FEVR cases did not undergo surgery.

Data collected from charts included gender, age presenting to our outpatient clinic, clinical presentation in each eye (stage, other ocular findings except RD), therapy procedure, referral diagnosis (if provided), preoperative diagnosis, family history, prior ocular surgery, premature birth, ocular trauma, ultra-widefield fundus image, diagnostic timing, year of initial clinic visit, year of FEVR diagnosis, patient FA. Patient FA and their parents FA were performed when available. There were some patients and parents did not undergo FA, because they were too young to cooperate with FA or were sensitive to fluorescein sodium.

According to the diagnostic timing, the 48 FEVR patients were divided into 2 groups: group 1, FEVR diagnosed preoperatively; group 2, FEVR diagnosed intra-/post-operatively. Patients, who were clearly identified with avascular peripheral retina and vascular anomalies consistent with FEVR before vitreoretinal or scleral buckling surgery in our department, were classified as FEVR diagnosed preoperatively. Patients, who had no evidence associated with FEVR preoperatively and were diagnosed as FEVR through intraoperative FA or impressing peripheral sclera during surgery, were classified as FEVR diagnosed intraoperatively. Patients, who were identified with avascular peripheral retina and vascular anomalies consistent with FEVR during postoperative follow-up, were classified as FEVR diagnosed postoperatively.

\section{Statistical analysis}

The data were analyzed using SPSS 24.0 software (SPSS, Inc., Chicago, IL, USA). Descriptive summary statistics were presented as mean and SD, as range and median for continuous variables, and as number and proportion for categorical data. The differences between the means were tested using t-test. The differences between the groups were tested using Chi-square test or Mann-Whitney U test. Risk factors for diagnostic timing were analyzed using binary logistic regression. Potential risk factors obtained from clinical charts were the patient's age at initial clinic visit (years), gender, preterm birth, ocular trauma, surgery in other hospital before first clinic visit, preoperative ocular 
manifestations, ocular involvement, clinical stage of patients at initial presentation, clinical stage in contralateral eye, year of diagnosis, year at initial clinic visit (years), wide-angle fundus imaging preoperatively, initial surgery in our hospital, father/mother's FA. These clinical factors were included in univariate analysis. A logistic multivariate regression forward stepwise model was constructed to identify independent risk factors from variables demonstrating statistically significant associations $(P<0.05)$ with risks of FEVR diagnosis preoperatively by univariate analysis.

\section{Results and case descriptions}

\section{Subjects}

Patient demographics are shown in Table 1. Thirteen females and 35 males were included in the study. The age of patients ranged from 3 months to 58 years and the median was 12 years. $47.9 \%$ of cases were diagnosed as FEVR preoperatively, while $52.1 \%$ cases were diagnosed as FEVR intra-/post-operatively. In the study, the stage of FEVR patient was defined as the stage of more severe eye. $95.8 \%$ of patients were advanced FEVR (stage 3-5), while the contralateral eyes of $81.2 \%$ of FEVR patients were mild FEVR (stage 1-2). Moreover, 95.8\% of the FEVR patients were diagnosed as non-specific retinopathy in other hospital or before surgery in our hospital, including unilateral RD (52.1\%), bilateral RD (8.3\%), unilateral PFV (4.2\%), bilateral PFV (4.2\%). In the cohort, the majority of patients were bilateral FEVR and only one patient was unilateral FEVR. The unilateral FEVR patient has a positive family history and her contralateral eye has no vascular abnormalities.

\section{Risk factors}

A total of 14 potential factors entered the univariate analysis, and 5 were found statistically associated with diagnosis of FEVR preoperatively (Table 2). In multivariate regression analysis, two factors were identified significantly associated with diagnosis of FEVR preoperatively: preoperative ocular manifestations (OR, 0.104; P, 0.022) and father/ mother's FA (OR, 0.105; P, 0.035). The patients who had $\mathrm{RD}$ only before surgery and the cases whose parents' FA were positive were more likely to be diagnosed as FEVR preoperatively. In contrary, the patients who had more complicated ocular manifestations and the cases who had no family history of FEVR were more susceptible to be diagnosed as FEVR intra-/post-operatively. Namely, these FEVR cases would be prone to be missed diagnosed preoperatively. The reasons of missed diagnosis of FEVR preoperatively were summarized in the Table 3 . In the study, the most common reason for missed diagnosis of FEVR preoperatively was that there was no ultra-wide-field scanning laser ophthalmoscopy at initial clinic visit or preoperatively.

\section{Case descriptions \\ Other ocular manifestations except $R D$ at the first clinic visit in our department}

Table 4 summarized the clinical characteristics of the complicated FEVR patients who had other ocular manifestation except RD at the first clinic visit. The expressivity of FEVR patients were greatly variable. The main clues for these complicated FEVR were listed in the Table 5.

\section{Complicated FEVR patients diagnosed as FEVR preoperatively}

In FEVR diagnosed preoperatively, positive family history was essential for FEVR patients with bilateral invisible fundus and was important for FEVR patients with unilateral invisible fundus. Patient no. 4 had only unilateral disappeared anterior chamber and positive family history, so that we diagnosed him as FEVR but not PFV. And in the operation, FA performed in the contralateral eye confirmed the diagnosis of FEVR (Fig. 1).

\section{Complicated FEVR patients diagnosed as FEVR intraoperatively}

Patient no. 5-16 were FEVR cases diagnosed during the surgery. We performed intraoperative FA examination under general anesthesia in the suspected FEVR patients $\leq 3$ years old (patient no. 5-11). These patients had only one eye involved seriously with invisible fundus, while the contralateral eye seemed like a healthy one (Fig. 2). Intraoperative FA could help us to diagnose FEVR correctly. Patient no. $12-16$ were patients $>3$ years old. We found avascular peripheral area and peripheral vascular anomalies when we impressed the peripheral sclera during the vitrectomy (Fig. 3).

\section{Complicated FEVR patients diagnosed as FEVR postoperatively}

Patient no. 17-21 were FEVR patients diagnosed during follow-up postoperatively. Patient no. 17 was misdiagnosed as unilateral PFV preoperatively due to the contralateral eye was completely normal. Considering the shallow anterior chamber, lensectomy was operated to reshape the anterior chamber. During the follow up, the fundus image of the surgical eye showed the radial retinal fold in which all major retinal vessels were involved, which indicating the patient might be FEVR (Fig. 4). Further, we executed FA examination in the patient no. 17 and her parents. Unfortunately, her parents' FA was positive. The patient's FA showed that the contralateral eye was completely normal and she was diagnosed as unilateral FEVR.

Patient no. 18-20 were all complicated with high myopia. The RD in these cases were prone to be diagnosed as non-specific RD. Both eyes of patient no. 18 underwent a photocoagulation therapy in the referral hospital, and the laser scars made FEVR diagnosis more difficult. 
Table 1 Demographic, ocular characteristics, referral or preoperative diagnosis of FEVR patients

\begin{tabular}{|c|c|c|c|c|}
\hline Variable & $\begin{array}{l}\text { Total } \\
(n=48)\end{array}$ & $\begin{array}{l}\text { FEVR diagnosed } \\
\text { pre-operatively } \\
(n=23)\end{array}$ & $\begin{array}{l}\text { FEVR diagnosed } \\
\text { intra-/post-operatively } \\
(n=25)\end{array}$ & $P$ value \\
\hline Age at initial clinic visit (years) & $14.22 \pm 13.87$ & $15.65 \pm 10.17$ & $12.90 \pm 16.68$ & $0.499^{*}$ \\
\hline Age range (years), (median) & $0.25-58(12)$ & $0.25-41(14)$ & $0.25-58(6)$ & \\
\hline \multicolumn{5}{|l|}{ Sex } \\
\hline Female & $13(27.1)$ & $4(17.4)$ & $9(36.0)$ & $0.147 \#$ \\
\hline Male & $35(72.9)$ & $19(82.6)$ & $16(64.0)$ & \\
\hline \multicolumn{5}{|l|}{$\begin{array}{l}\text { Clinical stage of patients at initial } \\
\text { presentation, } \mathrm{n}(\%) \#\end{array}$} \\
\hline Stage 2 & $2(4.2)$ & $0(0.0)$ & $2(8.0)$ & $0.398 \wedge$ \\
\hline Stage 3 & $11(22.9)$ & $7(30.4)$ & $4(16.0)$ & \\
\hline Stage 4 & $12(25.0)$ & $7(30.4)$ & $5(20.0)$ & \\
\hline Stage 5 & $23(47.9)$ & $9(39.1)$ & $14(56.0)$ & \\
\hline \multicolumn{5}{|l|}{$\begin{array}{l}\text { Clinical stage in contralateral } \\
\text { eye, } \mathrm{n}(\%)\end{array}$} \\
\hline NON-FEVR & $1(2.1)$ & $0(0.0)$ & $1(4.0)$ & $0.216 \wedge$ \\
\hline Stage 1 & $22(45.8)$ & $10(43.5)$ & $12(48.0)$ & \\
\hline Stage 2 & $17(35.4)$ & $7(30.4)$ & $10(40.0)$ & \\
\hline Stage 3 & $5(10.4)$ & $3(13.0)$ & $2(8.0)$ & \\
\hline Stage 4 & $1(2.1)$ & $1(4.4)$ & $0(0.0)$ & \\
\hline Stage 5 & $2(4.2)$ & $2(8.7)$ & $0(0.0)$ & \\
\hline \multicolumn{5}{|l|}{ Patient FA } \\
\hline+ & $35(72.9)$ & $15(65.2)$ & $20(80.0)$ & - \\
\hline N/A & $13(27.1)$ & $8(34.8)$ & $5(20.0)$ & \\
\hline \multicolumn{5}{|l|}{$\begin{array}{l}\text { Referral diagnosis/pre-operative } \\
\text { diagnosis, n (\%) }\end{array}$} \\
\hline Unilateral RD & $25(52.1)$ & $14(60.9)$ & $11(44.0)$ & - \\
\hline Bilateral RD & $4(8.3)$ & $3(13.0)$ & $1(4.0)$ & \\
\hline Bilateral PFV & $2(4.2)$ & $2(8.7)$ & $0(0.0)$ & \\
\hline Unilateral PFV & $4(8.3)$ & $1(4.3)$ & $3(12.0)$ & \\
\hline Ocular trauma & $1(2.1)$ & $1(4.3)$ & $0(0.0)$ & \\
\hline Bilateral FEVR & $2(4.2)$ & $2(8.7)$ & $0(0.0)$ & \\
\hline Unilateral PFV + ROP & $1(2.1)$ & $0(0.0)$ & $1(4.0)$ & \\
\hline Unilateral RB & $2(4.2)$ & $0(0.0)$ & $2(8.0)$ & \\
\hline $\begin{array}{l}\text { Unilateral congenital } \\
\text { cataract }+\mathrm{VH}+\mathrm{RD}\end{array}$ & $1(2.1)$ & $0(0.0)$ & $1(4.0)$ & \\
\hline Unilateral RD + VH & $1(2.1)$ & $0(0.0)$ & $1(4.0)$ & \\
\hline $\mathrm{VH}+$ contralateral ERM & $1(2.1)$ & $0(0.0)$ & $1(4.0)$ & \\
\hline Unilateral VH + aphakia & $1(2.1)$ & $0(0.0)$ & $1(4.0)$ & \\
\hline Bilateral MF $+\mathrm{LMH}+\mathrm{HM}$ & $1(2.1)$ & $0(0.0)$ & $1(4.0)$ & \\
\hline $\begin{array}{l}\text { Bilateral congenital cataract+ } \\
\text { unilateral PFV }\end{array}$ & $1(2.1)$ & $0(0.0)$ & $1(4.0)$ & \\
\hline Unilateral ERM & $1(2.1)$ & $0(0.0)$ & $1(4.0)$ & \\
\hline
\end{tabular}

$R D$ Retinal detachment, PFV Persistent fetal vasculature, ROP Retinopathy of prematurity, RB Retinoblastoma, $V H$ Vitreous hemorrhage, ERM Epiretinal membrane, MF Myopic foveoschisis, LMH Lamellar macular hole, HM High myopia

* t-test

\# Chi-square test

$\wedge$ Mann-Whitney U test 
Table 2 Univariate and Multivariate Logistic Regression Analysis of Risk Factors of diagnostic timing in FEVR patients

\begin{tabular}{|c|c|c|c|c|c|c|}
\hline \multirow[t]{2}{*}{ Risk factors } & \multirow{2}{*}{$\begin{array}{l}\text { FEVR diagnosed } \\
\text { pre-operatively } \\
(n=23)\end{array}$} & \multirow{2}{*}{$\begin{array}{l}\text { FEVR diagnosed } \\
\text { intra-/post-operatively } \\
(n=25)\end{array}$} & \multicolumn{2}{|l|}{ Univariate Analysis } & \multicolumn{2}{|l|}{ Multivariate Analysis } \\
\hline & & & OR $(95 \% \mathrm{Cl})$ & $P$ & $\mathrm{OR}(95 \% \mathrm{Cl})$ & $P$ \\
\hline \multicolumn{7}{|l|}{$\begin{array}{l}\text { Preoperative ocular manifestations, } \\
\text { n (\%) (no. of patients) }\end{array}$} \\
\hline RD only & 19 (82.6) & $8(32.0)$ & $0.099(0.025-0.389)$ & 0.001 & $0.104(0.015-0.725)$ & 0.022 \\
\hline $\begin{array}{l}\text { Other manifestations except/without } \\
\text { RD }\end{array}$ & $4(17.4)$ & $17(68.0)$ & & & & \\
\hline \multicolumn{7}{|l|}{ Father/mother FA, n (\%) } \\
\hline- & $2(8.7)$ & $9(36.0)$ & $0.141(0.023-0.857)$ & 0.033 & $0.105(0.013-0.855)$ & 0.035 \\
\hline+ & $11(47.8)$ & $7(28.0)$ & & & & \\
\hline N/A & $10(43.5)$ & $9(36.0)$ & & & & \\
\hline \multicolumn{7}{|l|}{ Year of diagnosis, n (\%) } \\
\hline 2010-2015 & $7(30.4)$ & $17(68.0)$ & $0.161(0.044-0.583)$ & 0.005 & & \\
\hline $2016-2018$ & $16(69.6)$ & $8(32.0)$ & & & & \\
\hline \multicolumn{7}{|l|}{ Year at initial clinic visit (years), n (\%) } \\
\hline 2007-2014 & $7(30.4)$ & $19(76.0)$ & $0.138(0.039-0.496)$ & 0.002 & & \\
\hline 2015-2018 & $16(69.6)$ & $6(24.0)$ & & & & \\
\hline \multicolumn{7}{|l|}{$\begin{array}{l}\text { Wide-angle fundus imaging } \\
\text { preoperatively, } \mathrm{n}(\%)\end{array}$} \\
\hline No & $6(26.1)$ & $20(80.0)$ & $0.088(0.023-0.341)$ & 0.000 & & \\
\hline Yes & $17(73.9)$ & $5(20.0)$ & & & & \\
\hline \multicolumn{7}{|l|}{ Ocular trauma, n (\%) } \\
\hline No & $17(73.9)$ & $24(96.0)$ & $0.118(0.013-1.072)$ & 0.058 & & \\
\hline Yes & $6(26.1)$ & $1(4.0)$ & & & & \\
\hline \multicolumn{7}{|l|}{$\begin{array}{l}\text { Clinical stage in contralateral } \\
\text { eye, } \mathrm{n}(\%)\end{array}$} \\
\hline Stage 1-Stage 2 & $17(73.9)$ & $23(92.0)$ & $4.059(0.728-22.637)$ & 0.110 & & \\
\hline Stage 3-Stage 5 & $6(26.1)$ & $2(8.0)$ & & & & \\
\hline \multicolumn{7}{|l|}{$\begin{array}{l}\text { Age at initial clinic visit (years), } \\
\mathrm{n}(\%)\end{array}$} \\
\hline $0.25-12$ & $10(43.5)$ & $16(64.0)$ & $0.433(0.136-1.381)$ & 0.157 & & \\
\hline $13-58$ & $13(56.5)$ & $9(36.0)$ & & & & \\
\hline \multicolumn{7}{|l|}{ Gender, n (\%) } \\
\hline Female & $4(17.4)$ & $9(36.0)$ & $0.374(0.097-1.447)$ & 0.154 & & \\
\hline Male & 19 (82.6) & $16(64.0)$ & & & & \\
\hline \multicolumn{7}{|l|}{ Preterm birth, n (\%) } \\
\hline Yes & $0(0.0)$ & $1(4.0)$ & $0.000(0.000-)$ & 1.000 & & \\
\hline No & $23(100.0)$ & $24(96.0)$ & & & & \\
\hline \multicolumn{7}{|l|}{$\begin{array}{l}\text { Surgery in other hospital before } \\
\text { first clinic visit, } \mathrm{n}(\%)\end{array}$} \\
\hline No & $21(91.3)$ & $19(76.0)$ & $3.316(0.596-18.451)$ & 0.171 & & \\
\hline Yes & $2(8.7)$ & $6(24.0)$ & & & & \\
\hline \multicolumn{7}{|l|}{ Ocular involvement, n (\%) } \\
\hline Bilateral & $6(26.1)$ & $5(20.0)$ & $0.908(0.253-3.251)$ & 0.882 & & \\
\hline Unilateral & $17(73.9)$ & $20(80.0)$ & & & & \\
\hline
\end{tabular}


Table 2 Univariate and Multivariate Logistic Regression Analysis of Risk Factors of diagnostic timing in FEVR patients (Continued)

\begin{tabular}{|c|c|c|c|c|c|c|}
\hline \multirow[t]{2}{*}{ Risk factors } & \multirow{2}{*}{$\begin{array}{l}\text { FEVR diagnosed } \\
\text { pre-operatively } \\
(n=23)\end{array}$} & \multirow{2}{*}{$\begin{array}{l}\text { FEVR diagnosed } \\
\text { intra-/post-operatively } \\
(n=25)\end{array}$} & \multicolumn{2}{|l|}{ Univariate Analysis } & \multicolumn{2}{|c|}{ Multivariate Analysis } \\
\hline & & & OR $(95 \% \mathrm{Cl})$ & $P$ & OR $(95 \% \mathrm{Cl})$ & $P$ \\
\hline Stage 1-Stage 4 & $14(60.9)$ & $11(44.0)$ & $1.980(0.626-6.259)$ & 0.245 & & \\
\hline Stage 5 & $9(39.1)$ & $14(56.0)$ & & & & \\
\hline \multicolumn{7}{|c|}{ Initial surgery in our hospital, n (\%) } \\
\hline $\mathrm{SB} / \mathrm{SE}$ & $7(30.4)$ & $5(20.0)$ & $1.750(0.466-6.568)$ & 0.407 & & \\
\hline$P P V / L$ & $16(69.6)$ & $20(80.0)$ & & & & \\
\hline
\end{tabular}

Cut-off value based on median value in the total sample of 48 patients

${ }^{a}$ The clinical stage of FEVR patients were defined as the clinical stage of severer eye

In our department, scleral buckling was performed in the RRD eye. In a month after the surgery, we found the peripheral avascular area over the scleral indentation zone. Then, FA examination confirmed the diagnosis of FEVR (Fig. 5).

\section{Discussion}

FEVR is a heterogeneous genetic disease. The manifestations of FEVR are greatly variable, which lead to the missed diagnosis of FEVR. Our results showed that FEVR patients who needed surgery might manifest with unilateral RD, bilateral $\mathrm{RD}$, disappearance of anterior chamber, retrolental membrane, ocular trauma, $\mathrm{VH}$, ERM, nystagmus, phthisis bulbi, LMH, MF, HM. All of the ocular presentations were so confusing that missed diagnosis of FEVR was easily made. There were also many case reports about FEVR being mistaken for

Table 3 The reasons of missed diagnosis of FEVR preoperatively

\begin{tabular}{|c|c|}
\hline $\begin{array}{l}\text { Reasons of missed diagnosis } \\
\text { of FEVR preoperatively }\end{array}$ & Cases $(n=28)^{a}$ \\
\hline $\begin{array}{l}\text { Age }<3 \text { years, unilateral leukocoria with invisible } \\
\text { fundus }+ \text { contralateral fundus seemingly normal }\end{array}$ & 8 \\
\hline $\begin{array}{l}\text { Age } \geq 3 \text { years, without ultra-wide-field scanning } \\
\text { laser ophthalmoscopy at initial clinic visit or } \\
\text { preoperatively }\end{array}$ & 9 \\
\hline $\begin{array}{l}\text { Bilateral vitreoretinal surgery or peripheral retinal } \\
\text { photocoagulation in other hospital before the } \\
\text { first clinic visit }\end{array}$ & 2 \\
\hline Unilateral VH + contralateral phthisis bulbi & 2 \\
\hline Unilateral VH + contralateral ERM & 1 \\
\hline Unilateral RD + contralateral ERM & 1 \\
\hline Unilateral ERM & 1 \\
\hline $\begin{array}{l}\text { Bilateral pathological myopia + myopic } \\
\text { foveoschisis + lamellar macular hole }\end{array}$ & 1 \\
\hline $\begin{array}{l}3 \text { years < age }<4 \text { years, poor cooperation, } \\
\text { ultra-wide-field scanning laser ophthalmoscopy } \\
\text { failed to capture the peripheral retina }\end{array}$ & 1 \\
\hline Premature birth history & 1 \\
\hline Ocular trauma & 1 \\
\hline
\end{tabular}

${ }^{a}$ The number of cases in Table 3 is totally 28 , which is more than the number of FEVR patients diagnosed intra-/post-operatively $(n=25)$. Because some patients had multiple reasons of missed diagnosis of FEVR preoperatively other vitreoretinopathies, including PFV, VH, ROP, Rb, full-thickness macular hole (FTMH), which strengthened our findings [12-19]. The presentation of FEVR may mimic the presentation of other pediatric and adult vitreoretinopathies, thus careful examination of peripheral retina and avascular peripheral zone is often essential in making the diagnosis of FEVR.

In the study, risk factors analysis showed that the patients who had RD only were more likely to be diagnosed as FEVR preoperatively, while the patients who had other ocular manifestations except RD were more likely to be diagnosed as FEVR intra-/post-operatively. Because the rate of RD in FEVR patients is relatively high. It is reported that RD occurred in $20 \%$ eyes with FEVR and $47.6 \%$ nonsyndromic congenital RD patients were FEVR patients $[7,20]$. In addition, the types of RD in FEVR patients were very variable, such as RRD, falciform retinal folds, PVR with formation of a retrolental membrane and exudative retinal detachment (ERD). And the undetached fellow eyes of FEVR-RRD patients were generally characterized by vascular leakage, lattice degeneration, vitreous traction in the peripheral retina [5]. Considering the high rate of RD in FEVR, when we received a RD patient, we would spontaneously think of the possibility of FEVR. And, carefully examination of peripheral retina in the contralateral eyes of $\mathrm{RD}$ patients could be benefit to find FEVR. Bilateral dilated fundoscopy is mandatory and the clear image of peripheral retina is critical. Moreover, parent's FA is also an important clue which contributes to diagnose FEVR earlier, especially for patients with bilateral disappeared anterior chamber and invisible fundus. In this condition, we should perform FA in his/her parents preoperatively to confirm FEVR. Although the positive family history is not a prerequisite for FEVR, a positive family history is greatly helpful.

In addition, it was noteworthy that there were more patients diagnosed as FEVR preoperatively in recent 3 years than the first 6 years before 2016 (Table 2). First, this was due to the extensive application of ultra-widefield scanning laser ophthalmoscope (UWF 


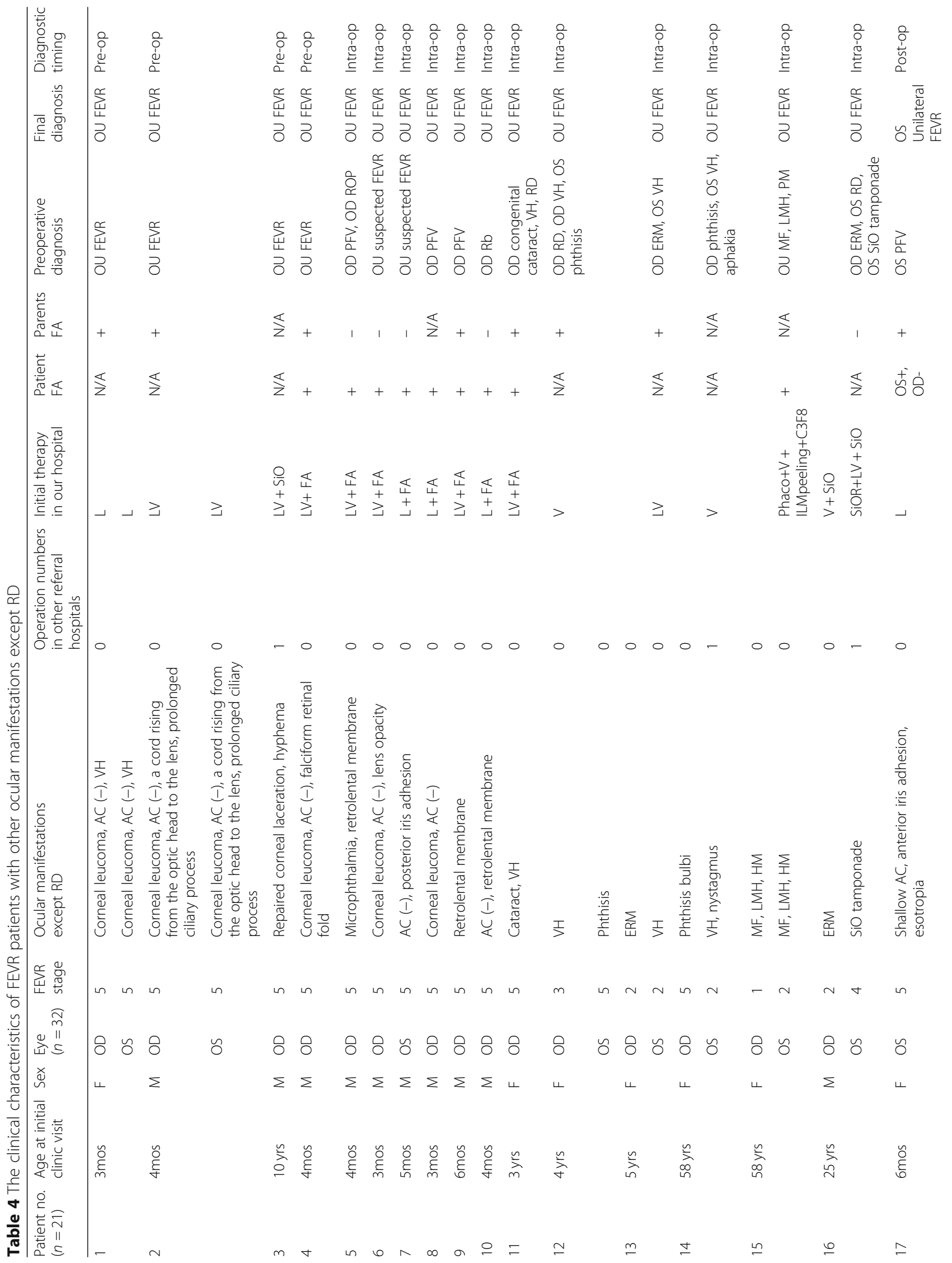




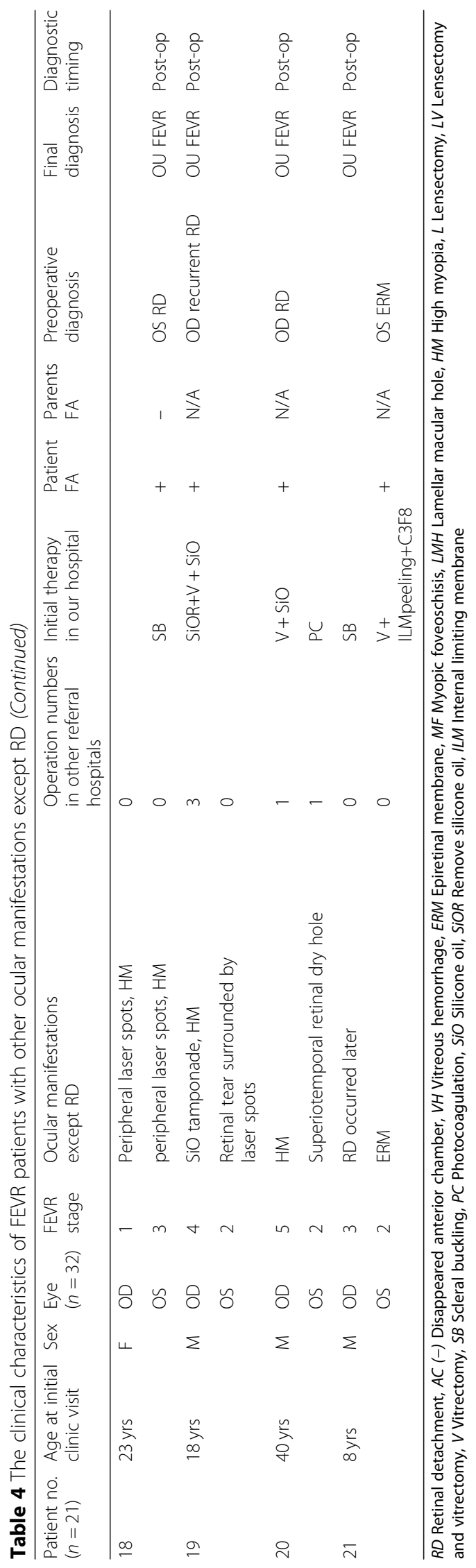


Table 5 The main clues for these complicated FEVR

\begin{tabular}{|c|c|}
\hline Main clue for diagnosing FEVR & Cases $(n=21)$ \\
\hline \multicolumn{2}{|l|}{$\begin{array}{l}\text { Complicated FEVR patients diagnosed } \\
\text { as FEVR preoperatively }\end{array}$} \\
\hline Family history (+) & 3 \\
\hline $\begin{array}{l}\text { Avascular peripheral area and peripheral } \\
\text { vascular anomalies in the contralateral eye }\end{array}$ & 1 \\
\hline \multicolumn{2}{|l|}{$\begin{array}{l}\text { Complicated FEVR patients diagnosed as } \\
\text { FEVR intraoperatively }\end{array}$} \\
\hline Intraoperative FA (+) in the contralateral eye & 7 \\
\hline $\begin{array}{l}\text { Avascular peripheral area and peripheral vascular } \\
\text { anomalies were found when the peripheral sclera } \\
\text { was impressed during the operation }\end{array}$ & 5 \\
\hline \multicolumn{2}{|l|}{$\begin{array}{l}\text { Complicated FEVR patients diagnosed as FEVR } \\
\text { postoperatively }\end{array}$} \\
\hline $\begin{array}{l}\text { Radial retinal fold involved in all major retinal vessels } \\
\text { during the follow-up }\end{array}$ & 1 \\
\hline $\begin{array}{l}\text { Peripheral avascular zone was found during the } \\
\text { follow-up }\end{array}$ & 4 \\
\hline
\end{tabular}

SLO) in our department in recent 3 years. UWF SLO could provide a $200^{\circ}$ photographic view of a fundus, and it was a valuable imaging tool for detecting fundus anomalies, especially the peripheral retina. It was reported that UWF SLO could assist in the diagnosis and evaluation of early-stage FEVR [21]. In the study, the most common reason for missed diagnosis of FEVR preoperatively was that there was no ultrawide-field scanning laser ophthalmoscopy at initial clinic visit or preoperatively. (Table 3) Second, over time, our understanding of FEVR was deeper and clinical experience was more abundant, and more and more patients could be accurately diagnosed as FEVR before surgery. The longitudinal changes in the diagnosis and management of FEVR over the years might have introduced bias into risk factor analysis. Thus, in the study, wide-angle fundus imaging preoperatively and year of diagnosis were not independent risk factors of diagnostic timing in the FEVR patients. More cases would be required for further confirmation.

However, when FEVR complicated with other ocular presentations, the diagnosis of FEVR would still be quite challenging. In the study, we concluded procedures for diagnosing FEVR earlier. For patients with unilateral RD only, careful examination of the contralateral eye was essential to find peripheral vascular anomalies especially in adolescent. For patients with bilateral RD only, we should be more alert to the possibility of FEVR and it was necessary to inspect the peripheral retinal vessel with ultra-widefield retinal image. Ultra-widefield fundus photography was important in the management of pediatric retinal diseases and could aid the physician in the documentation and evaluation of peripheral retinal pathology [22]. For pediatric patients with unilateral disappeared anterior chamber, careful examination of peripheral retina of the contralateral eye was critical and parent's FA could help us to differentiate FEVR with PFV. If parent's FA is negative, we should perform FA for the pediatric patient who could not cooperate with examination during surgery under general anesthesia. For pediatric patients with bilateral disappeared anterior chamber, we performed FA in their parents. If the parents' FA were positive, the diagnosis of FEVR could be made preoperatively. In addition, for patients with bilateral ocular fundus disorders except RD without any systemic disorders, we should make the distinction between FEVR and non-specific vitreoretinopathies. During the surgery, observing peripheral retina with simultaneous scleral impression could avoid the missed diagnosis of FEVR, especially for suspected FEVR cases preoperatively. We wished that the procedures would help clinicians diagnose FEVR earlier.
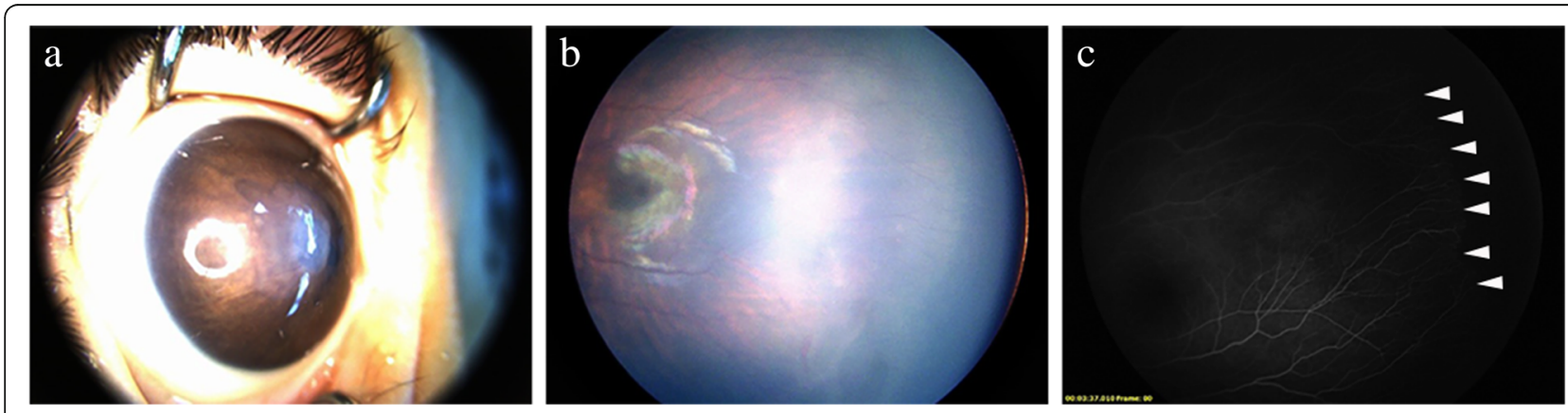

Fig. 1 Anterior segment photograph and fundus image in patient no. 4. a The anterior chamber of right eye was disappeared and the central cornea was edema. The fundus of right eye was absolutely invisible. $\mathbf{b}$ The fundus image of left eye by Retcam showed that there were peripheral avascular zone and supernumerous ramification of vascular. c The angiogram of left eye indicated that there was avascular area in peripheral retina, increased ramification of vessel (arrowhead). The FA image confirmed the diagnosis of FEVR 

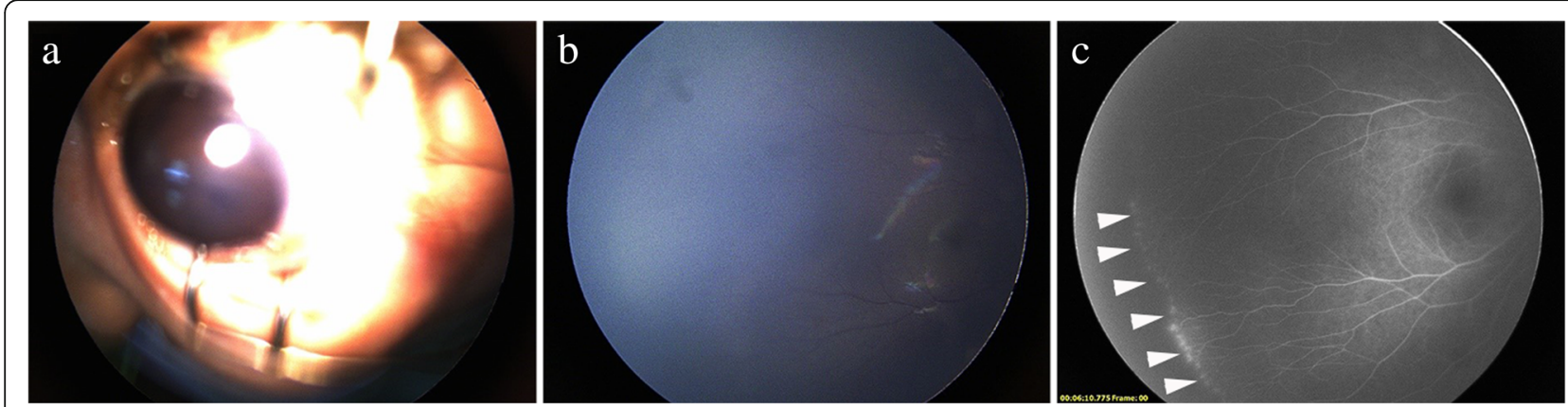

Fig. 2 Anterior segment photograph and fundus image in patient no. 7. a the anterior chamber of left eye was disappeared and posterior iris was adhered. The fundus of left eye was absolutely invisible. $\mathbf{b}$ the fundus image of right eye by Retcam showed that the peripheral retina was not clear and the fundus seemed likely to be normal. c FA was performed during the surgery under general anesthesia. The angiogram of right eye suggested that there was avascular area, increased ramification of vessel and bulb-like or telangiectatic endings in the periphery (arrowhead)

Limitations of the study were as follows: this was a retrospective clinical study; the number of cases was small since FEVR is a relatively uncommon disease; in addition, genetic sequencing was not listed in the study. Because genetic sequencing was generally too expensive to some families in the study. And some parents did not undergo FA examination due to their absence to our clinic or sensitivity to fluorescein sodium. However, not all FEVR patients have FEVR gene mutations and positive family history. Rao FQ et.al reported that $38.7 \%$ Chinese patients with FEVR were confirmed to harbor mutations in the six known disease-causing genes [23]. Kashani et.al reported that, $43 \%$ FEVR patients had detectable mutations in FZD4, NDP, or TSPAN12 in their study cohort, while

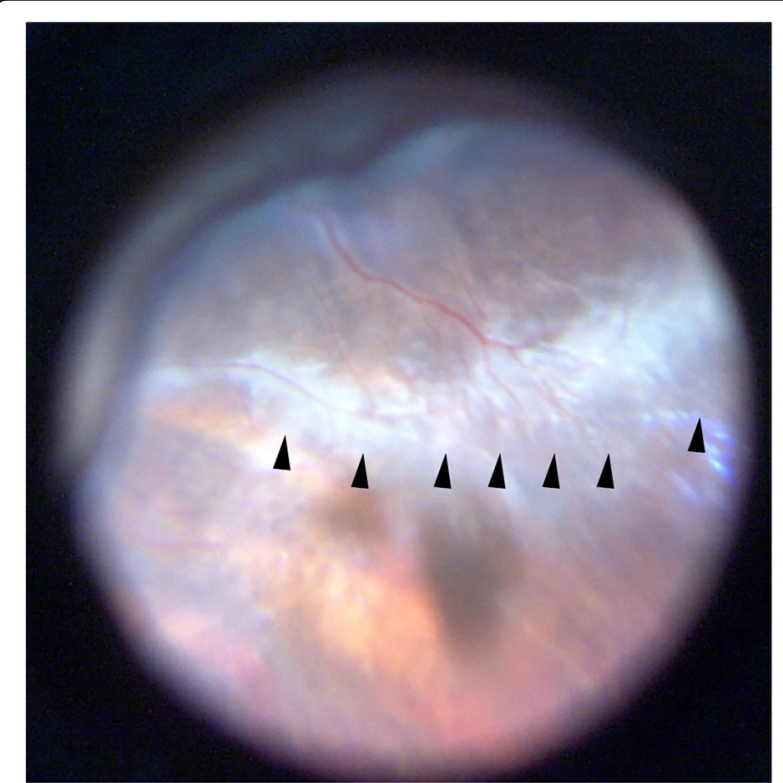

Fig. 3 The intraoperative fundus image of the right eye in patient no.12. During the pars plana vitrectomy, the peripheral avascular zone was found when the sclera was impressed (arrowhead) only $8 \%$ FEVR patients reported a positive family history of FEVR in a first-degree relative [24]. Thus, Genetic sequencing and family history is not a prerequisite for FEVR diagnosis. Namely, the lack of genetic sequencing and family history did not influence our correct diagnosis of FEVR, although a positive family history could contribute to diagnose FEVR earlier. The diagnosis of FEVR was ultimately based on clinical findings.

\section{Conclusions}

In summary, the phenotype of FEVR was greatly variable, they can disguise as many non-specific vitreoretinopathies. The most non-specific referral diagnosis or pre-operative diagnosis was unilateral $\mathrm{RD}$, bilateral $\mathrm{RD}$, unilateral PFV, bilateral PFV. Furthermore, the more complicated the patient's manifestation, the more delayed the diagnosis of FEVR will be. A positive family history or a simple ocular presentation with RD only

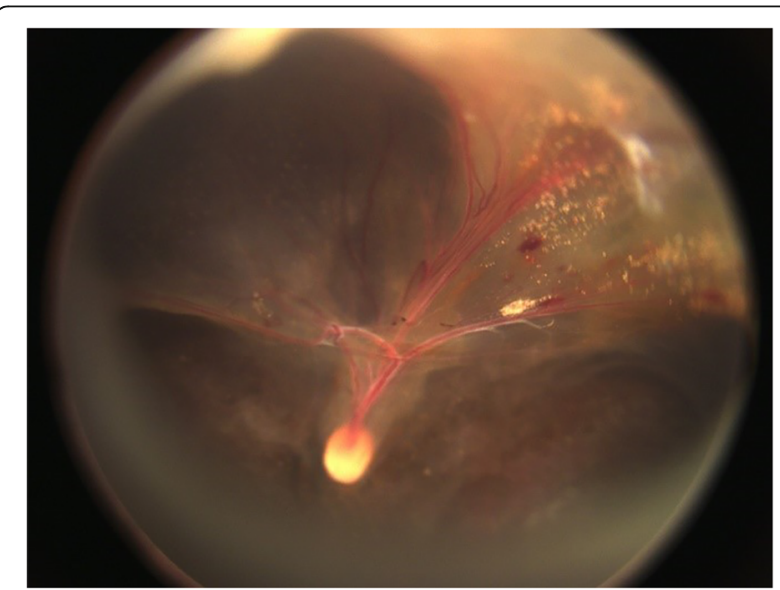

Fig. 4 The postoperative fundus image of the left eye in patient no. 17. The radial retinal fold involving all major retinal vessels indicated that the patient might be a FEVR case 

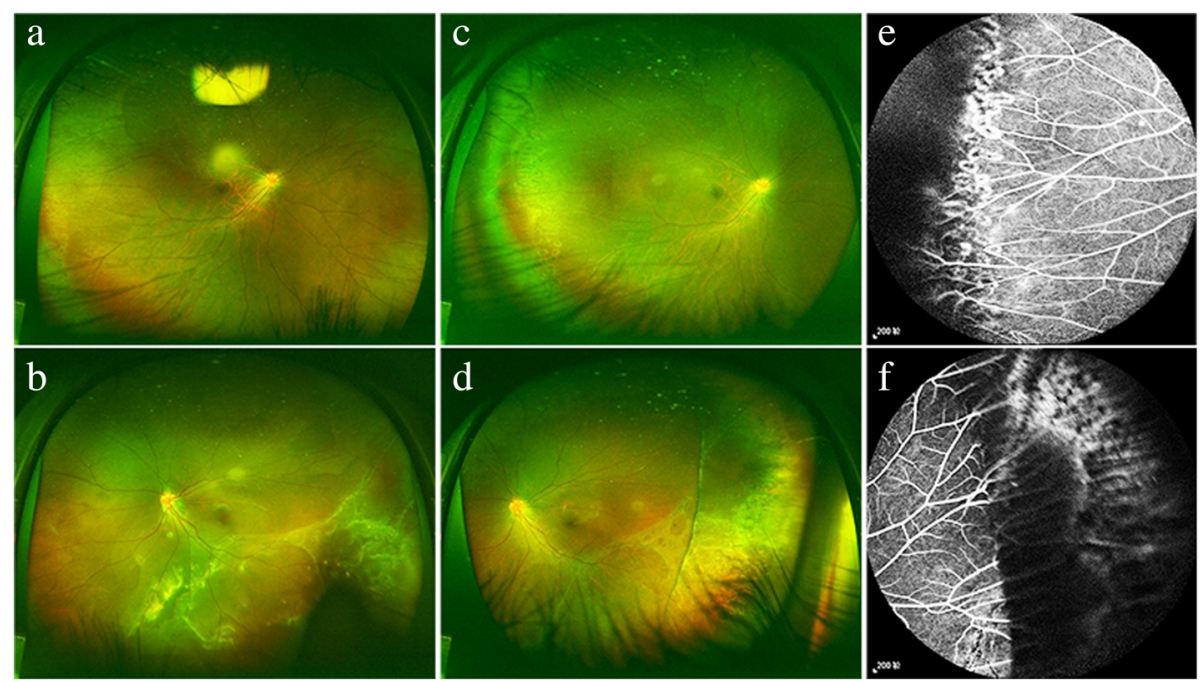

Fig. 5 The ultra-widefield fundus image and angiograms of patient no. 18. a and $\mathbf{b}$ The fundus image of the patient first presentation to our clinic. It showed that there were some laser spots in the periphery of the right eye and RD in the temporal and inferior of the left eye. $\mathbf{c}$ and $\mathbf{d}$ The fundus image of the patient in 1 month after scleral buckling surgery in our department. The peripheral avascular zones of both eyes were found. $\mathbf{e}$ and $\mathbf{f}$ The angiograms of the patient showed plenty of vascular ramifications and laser spots in both eye and impressed ridge in the left eye

could contribute to diagnose FEVR preoperatively. Carefully examination of the contralateral asymptomatic eye and FA in parents preoperatively might be helpful to diagnose complicated FEVR earlier. Patient's FA during the operation and careful inspection of the peripheral area of retina intraoperatively might prevent missed diagnosis of FEVR in suspected cases.

\section{Abbreviations}

AC: Anterior chamber; ERM: Epiretinal membrane; FEVR: Family exudative vitreoretinopathy; FTMH: Full-thickness macular hole; HM: High myopia; ILM: Internal limiting membrane; L: Lensectomy; LMH: Lamellar macular hole; LV: Lensectomy and vitrectomy; MF: Myopic foveoschisis;

PC: Photocoagulation; PFV: Persistent fetal vasculature; RB: Retinoblastoma; RD: Retinal detachment; ROP: Retinopathy of prematurity; SB: Scleral buckling; SiO: Silicone oil; SiOR: Remove silicone oil; V: Vitrectomy; $\mathrm{VH}$ : Vitreous hemorrhage

\section{Acknowledgements}

The authors thank all the patients and their families for their participation.

\section{Authors' contributions}

Design and conduct of the study (FJX, PQZ); collection, analysis and interpretation of data (FJX, JL, PF); preparation of manuscript (FJX); critical review and final approval of the manuscript (PQZ). All authors read and approved the final manuscript.

\section{Funding}

This study was supported by grants from the Science and Technology Commission of Shanghai Municipality (17411952900), Shanghai Shen Kang Hospital Development Center Program (16CR4017A), the National Natural Science Foundation Project of China (81770964, 81470642), and the Program for Shanghai Outstanding Academic Leader.

\section{Availability of data and materials}

The datasets used and/or analyzed during the current study available from the corresponding author on reasonable request.

\section{Ethics approval and consent to participate}

This study was approved by Ethics Committee of Xin Hua Hospital affiliated to Shanghai Jiao Tong University School of Medicine. All procedures performed in studies involving human participants were in accordance with the ethical standards of the institutional and/or national research committee and with the 1964 Helsinki Declaration and its later amendments or comparable ethical standards. The written informed consent to participate was obtained from each patient or his/her legal guardians included in the study.

\section{Consent for publication}

The written informed consent was obtained from all individual participants to publish the study. For patients under 18-year old, the written consent was obtained from his/her legal guardians. The written consent for publication of the anterior segment photographs, fundus images and angiograms were obtained from the participants or his/her legal guardians.

\section{Competing interests}

The authors declare that they have no competing interests.

Received: 4 December 2018 Accepted: 20 May 2019

Published online: 08 June 2019

\section{References}

1. Gilmour DF. Familial exudative vitreoretinopathy and related retinopathies. Eye (Lond). 2015;29(1):1-14

2. Miyakubo $\mathrm{H}$, Hashimoto K, Miyakubo S. Retinal vascular pattern in familial exudative vitreoretinopathy. Ophthalmology. 1984;91(12):1524-30.

3. Pendergast SD, Trese MT. Familial exudative vitreoretinopathy. Results of surgical management. Ophthalmology. 1998;105(6):1015-23.

4. Ranchod TM, Ho LY, Drenser KA, Capone A Jr, Trese MT. Clinical presentation of familial exudative vitreoretinopathy. Ophthalmology. 2011; 118(10):2070-5.

5. Yuan M, Ding X, Yang Y, Liu F, Li J, Liang X, et al. Clinical features of affected and undetached fellow eyes in patients with fevr-associated rhegmatogenous retinal detachment. Retina. 2017:37(3):585-91.

6. Fei P, Yang W, Zhang Q, Jin H, Li J, Zhao P. Surgical management of advanced familial exudative vitreoretinopathy with complications. Retina. 2016;36(8):1480-5

7. van Nouhuys CE. Juvenile retinal detachment as a complication of familial exudative vitreoretinopathy. Fortschr Ophthalmol. 1989;86(3):221-3. 
8. Kashani AH, Brown KT, Chang E, Drenser KA, Capone A, Trese MT. Diversity of retinal vascular anomalies in patients with familial exudative vitreoretinopathy. Ophthalmology. 2014;121(11):2220-7.

9. Hocaoglu M, Karacorlu M, Sayman Muslubas I, Ersoz MG, Arf S. Anatomical and functional outcomes following vitrectomy for advanced familial exudative vitreoretinopathy: a single surgeon's experience. $\mathrm{Br} J$ Ophthalmol. 2017;101(7):946-50.

10. Ikeda T, Fujikado T, Tano Y, Tsujikawa K, Koizumi K, Sawa H, et al. Vitrectomy for rhegmatogenous or tractional retinal detachment with familial exudative vitreoretinopathy. Ophthalmology. 1999;106(6):1081-5.

11. Yamane T, Yokoi T, Nakayama Y, Nishina S, Azuma N. Surgical outcomes of progressive tractional retinal detachment associated with familial exudative vitreoretinopathy. Am J Ophthalmol. 2014;158(5):1049-55.

12. Kartchner JZ, Hartnett ME. Familial exudative vitreoretinopathy presentation as persistent fetal vasculature. Am J Ophthalmol Case Rep. 2017;6:15-7.

13. Robitaille JM, Wallace K, Zheng B, Beis MJ, Samuels M, Hoskin-Mott A, et al. Phenotypic overlap of familial exudative vitreoretinopathy (FEVR) with persistent fetal vasculature (PFV) caused by FZD4 mutations in two distinct pedigrees. Ophthalmic Genet. 2009;30(1):23-30.

14. Benoist D'azy C, Bonnin N, Maurin C, Farguette F, Chiambaretta F. Vitreous hemorrhage as the initial manifestation of familial exudative vitreoretinopathy in an eight-year-old child. J Fr Ophtalmol. 2016;39(6):549-53.

15. Tagami M, Kusuhara S, Honda S, Tsukahara Y, Negi A. Rapid regression of retinal hemorrhage and neovascularization in a case of familial exudative vitreoretinopathy treated with intravitreal bevacizumab. Graefes Arch Clin Exp Ophthalmol. 2008:246(12):1787-9.

16. John VJ, McClintic Jl, Hess DJ, Berrocal AM. Retinopathy of prematurity versus familial exudative vitreoretinopathy: report on clinical and angiographic findings. Ophthalmic Surg Lasers Imaging Retina. 2016;47(1):14-9.

17. Gologorsky D, Chang JS, Hess DJ, Berrocal AM. Familial exudative vitreoretinopathy in a premature child. Ophthalmic Surg Lasers Imaging Retina. 2013;44(6):603-5.

18. Ghassemi F, Bazvand F, Makateb A. Lesions simulating retinoblastoma at a tertiary care center. J Ophthalmic Vis Res. 2015;10(3):316-9.

19. Khwarg JW, Bourla D, Gonzales CA, Schwartz SD. Familial exudative vitreoretinopathy and macular hole exhibited in same individual. Semin Ophthalmol. 2007;22(2):85-6

20. Keser V, Khan A, Siddiqui S, Lopez I, Ren H, Qamar R, et al. The genetic causes of nonsyndromic congenital retinal detachment: a genetic and phenotypic study of Pakistani families. Invest Ophthalmol Vis Sci. 2017;58(2):1028-36.

21. Lyu J, Zhang Q, Wang SY, Chen YY, Xu Y, Zhao PQ. Ultra-wide-field scanning laser ophthalmoscopy assists in the clinical detection and evaluation of asymptomatic early-stage familial exudative vitreoretinopathy. Graefes Arch Clin Exp Ophthalmol. 2017:255(1):39-47.

22. Kang KB, Wessel MM, Tong J, D'Amico DJ, Chan RV. Ultra-widefield imaging for the management of pediatric retinal diseases. J Pediatr Ophthalmol Strabismus. 2013;50(5):282-8.

23. Rao FQ, Cai XB, Cheng FF, Cheng W, Fang XL, Li N, et al. Mutations in LRP5,FZD4, TSPAN12, NDP, ZNF408, or KIF11 genes account for 38.7\% of Chinese patients with familial exudative vitreoretinopathy. Invest Ophthalmol Vis Sci. 2017;58(5):2623-9.

24. Kashani AH, Learned D, Nudleman E, Drenser KA, Capone A, Trese MT. High prevalence of peripheral retinal vascular anomalies in family members of patients with familial exudative vitreoretinopathy. Ophthalmology. 2014; 121(1):262-8.

\section{Publisher's Note}

Springer Nature remains neutral with regard to jurisdictional claims in published maps and institutional affiliations.

Ready to submit your research? Choose BMC and benefit from:

- fast, convenient online submission

- thorough peer review by experienced researchers in your field

- rapid publication on acceptance

- support for research data, including large and complex data types

- gold Open Access which fosters wider collaboration and increased citations

- maximum visibility for your research: over $100 \mathrm{M}$ website views per year

At $\mathrm{BMC}$, research is always in progress.

Learn more biomedcentral.com/submissions 